\title{
A little help from residual $\beta$ cells has long-lasting clinical benefits
}

\author{
Anna Lam, ${ }^{1}$ Colin Dayan, ${ }^{2}$ and Kevan C. Herold ${ }^{3}$ \\ ${ }^{1}$ University of Alberta, Edmonton, Alberta, Canada. ${ }^{2}$ Cardiff University School of Medicine, Cardiff, United Kingdom. ${ }^{3}$ Yale University, New Haven, Connecticut, USA.
}

\begin{abstract}
Following type 1 diabetes (T1D) diagnosis, declining C-peptide levels reflect deteriorating $\beta$ cell function. However, the precise C-peptide levels that indicate protection from severe hypoglycemia remain unknown. In this issue of the $\mathrm{JCl}$, Gubitosi-Klug et al. studied participants from the landmark and ongoing Diabetes Control and Complications Trial (DCCT) and the Epidemiology of Diabetes Interventions and Complications (EDIC) study that had long-standing (about 35 years) T1D. The authors correlated severe hypoglycemia and other disease outcomes with residual C-peptide levels. While C-peptide secretion failed to associate with hemoglobin A1c (HbA1c) or microvascular complications, C-peptide levels greater than $0.03 \mathrm{nmol} / \mathrm{L}$ were linked with fewer episodes of severe hypoglycemia. These findings suggest that efforts to preserve finite $\beta$ cell function early in T1D can have meaningful, long-standing health benefits for patients.
\end{abstract}

\section{$\beta$ Cell function after type 1 diabetes diagnosis}

Despite major advances in insulin delivery and glucose monitoring, less than $30 \%$ of children and adults with type 1 diabetes (T1D) achieve levels of glucose control sufficient to prevent long-term complications (1). Higher proportions of the population are able to achieve optimal glycemic control in the first year after diagnosis, with gradual deterioration over the subsequent 5 years (2). This deterioration parallels the loss of endogenous $\beta$ cell function. More than $80 \%$ of individuals maintain a peak C-peptide response (greater than 0.20 $\mathrm{nmol} / \mathrm{L}$ ) at the end of the first year after diagnosis, compared with approximately $30 \%$ after four years, and even fewer with longer duration (3). Major efforts to develop immune therapies to preserve $\beta$ cell function in T1D have thus been made under the assumption that preserving some $\beta$ cell function would allow more people to achieve glycemic control targets and reduce hypoglycemia and the risk of long-term complications. But how much $\mathrm{C}$-peptide is required for clinical benefit, and how long does the effect last?

\section{Long-duration T1D outcomes}

In this issue of the JCI, Gubitosi-Klug et al. contributed important information toward answering the question of how C-peptide associates with long-term outcomes in T1D (4). Gubitosi-Klug et al. recalled patients from the landmark Diabetes Control and Complications Trial and the Epidemiology of Diabetes Interventions and Complications (DCCT/EDIC) study, now with an average 35-year duration of T1D, and assessed residual $\beta$ cell function by measuring $\mathrm{C}$-peptide during a mixedmeal tolerance test (MMTT). A total of 944 individuals from the original cohort $(\sim 63 \%)$ were investigated. As expected, the majority (827 individuals [88\%]) had no detectable C-peptide, even by ultrasensitive C-peptide assay. Low C-peptide

\section{Related Article: https://doi.org/10.1172/JCl143011}

Conflict of interest: The authors have declared that no conflict of interest exists.

levels were anticipated because of the long duration of disease but also because the DCCT entry criteria excluded individuals who had stimulated C-peptide of more than $0.50 \mathrm{nmol} / \mathrm{L}$ at entry or of more than $0.20 \mathrm{nmol} / \mathrm{L}$ if they were more than 5 years from diagnosis at entry (5). Nonetheless, $12.4 \%$ of recalled participants had detectable C-peptide after 35 years, and they were categorized into three groups: 11 (1.1\%) had high peak C-peptide $(>0.20$ $\mathrm{nmol} / \mathrm{L}), 60$ (6.5\%) had intermediate levels (0.03-0.200 nmol/L), and 46 (4.8\%) had low levels (0.003-0.03 nmol/L). Severe hypoglycemia rates were lower with intermediate $(48 \%)$ or high C-peptide (27\%) compared with low $(74 \%)$ or no (70\%) C-peptide, but there were no differences in hemoglobin A1c (HbA1c) or microvascular complication rates (4).

The observation that participants in the high C-peptide (>0.20 nmol/L) group after 35 years of T1D had similar HbA1c levels and long-term complications as their low/moderate counterparts (4) at first glance appears at odds with earlier DCCT reports. Early data showed that having peak C-peptide of more than $0.20 \mathrm{nmol} / \mathrm{L}$ coincided with improved $\mathrm{HbA1c}$, reduced risk of retinopathy progression, and less severe hypoglycemia (6-8). There are several possible reasons for these incongruent data. First, the insulin responses (indicated by C-peptide) in these follow-up participants were still modest when compared with those earlier in the course of T1D (4). The peak C-peptide levels were three times lower than in newly diagnosed T1D and nine times lower compared with nondiabetic controls (9). Second, in addition to the quantitative impairment, the participants showed abnormal C-peptide response kinetics (4). Dysfunctional secretion kinetics may also contribute to reduced effectiveness of secreted insulin. There is a disproportionate importance of first-phase insulin (10 minute) release in controlling postprandial hyperglycemia, which is lost early in T1D (9-12) and low- 


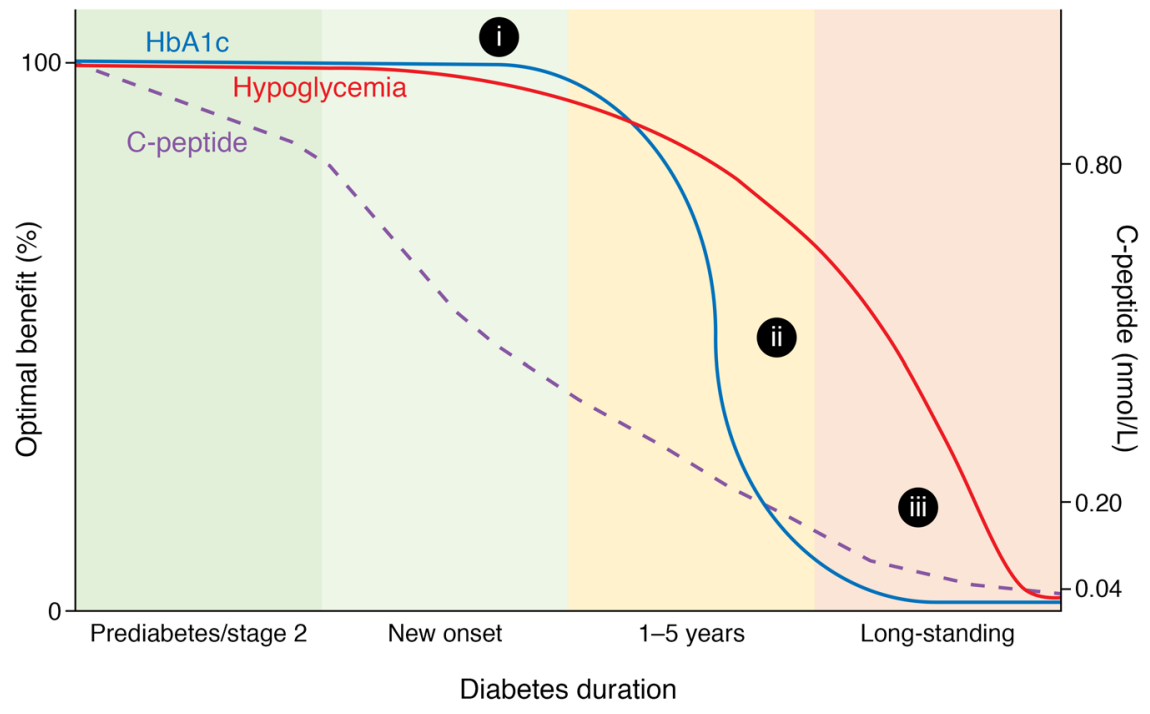

Figure 1. Putative relationship between clinical parameters for individuals with type 1 diabetes and C-peptide levels over the period that $\beta$ cell function declines. Optimal benefit reflects clinical benefit as a theoretical scale of $0 \%-100 \%$. C-peptide values were derived from clinical data, which generally include oral glucose tolerance tests for those in prediabetes/stage 2 diabetes and mixed-meal tolerance tests for those after diagnosis (stage 3 ). The levels may be summarized as more than 0.80 $\mathrm{nmol} / \mathrm{L}$ in prediabetes/stage 2 diabetes, $0.20-0.80 \mathrm{nmol} / \mathrm{L}$ in new-onset diabetes, $0.04-0.20 \mathrm{nmol} / \mathrm{L}$ 1-5 years after diagnosis, and less than $0.04 \mathrm{nmol} / \mathrm{L}$ in long-standing diabetes. The loss of C-peptide over a wide range associates with changes in hypoglycemia frequency, but the relationship with HbA1c levels flattens at high and low C-peptide levels. (i) In some individuals during prediabetes/ stage 2 diabetes, adequate C-peptide response and skilled insulin adjustments buffer HbA1c levels to hyperglycemia. Insulin release and lower C-peptide levels do not markedly affect hyperglycemia. (ii) In the first five years following T1D diagnosis, declining C-peptide values coincide with declining HbA1c levels, particularly among individuals with less effective self-management, adolescents, and young adults. (iii) Gubitosi-Klug et al. (4) demonstrated that after long-standing T1D, even small amounts of C-peptide provide protection against hypoglycemia.

ered in type 2 diabetes (ref. 13). GubitosiKlug and colleagues (4) observed evidence of dysfunctional secretion where the time to peak C-peptide levels was at 120 minutes for most of the participants, whereas, in healthy controls, the median time of peak C-peptide is 30 minutes. A comparison between the patterns of insulin secretion at this time and at an earlier stage of disease was not made. Third, the high C-peptide responders were more overweight (BMI 32.3 vs. $29 \mathrm{~kg} / \mathrm{m}^{2}$ for the other groups), with substantially higher insulin requirements (0.96 vs. $0.61-0.70$ units $/ \mathrm{kg} / \mathrm{d}$ in the other groups; $P<0.001$ ), suggestive of insulin resistance. Higher BMI has been associated with higher C-peptide levels at diagnosis (14), and this was true for the high C-peptide group (4). Insulin resistance might explain why, despite retaining and receiving more insulin, the high C-peptide group showed no difference in HbA1c compared with other groups and, in turn, no benefit in terms of microvascular complications. Finally, the number of subjects in this group was low ( $n$ $=11$ ), reducing the power to detect smaller differences in outcomes.

\section{Establishing C-peptide thresholds}

Gubitosi-Klug et al. (4) drew attention to a wider question that is key to the success of drug development in this field: How much C-peptide preservation is required for clinical benefit? The intrinsic relationship between endogenous C-peptide production and glycemic control is best observed in islet transplantation where restoration of $\beta$ cell function improves glycemic control, prevents hypoglycemia, and potentially slows progression of microvascular complications (15). Strong and continuous associations between stimulated C-peptide and time in range, glycemic variability (GV), and hypoglycemia have been demonstrated (16). However, the level of islet function needed to improve each differs; whereas excellent graft function is required to reduce hyperglycemia and GV, only a min- imal level of graft function is required to prevent hypoglycemia (17). Establishing clear thresholds for clinical outcomes outside of islet transplant has proven more difficult. Studies in recent-onset T1D $(<5$ years) typically include children and young adults and have shown an association between C-peptide and lower HbA1c (1821). In longer-duration diabetes ( $>5$ years, range 5-25 years), residual C-peptide has been associated with lower insulin requirements $(22,23)$. Notably, only stimulated C-peptide levels of more than 0.40 $\mathrm{nmol} / \mathrm{L}$ (two times greater than the threshold for eligibility in the study by GubitosiKlug et al.) have been linked with lower HbA1c (22). As in islet transplantation, low levels of C-peptide (as low as 0.04 nmo1/L in children 3-6 years from diagnosis and median random C-peptide of 0.032 $\mathrm{nmol} / \mathrm{L}$ in adults $15-25$ years from diagnosis) generally appear sufficient for protection against hypoglycemia $(19,20,23$, 24). Evidence from interventional studies comes from trials of successful immune interventions within 1-2 years after diagnosis with C-peptide levels 1.9-4 times higher than in the high responders described by Gubitosi-Klug et al. (4). Where C-peptide was preserved, these studies consistently showed a reduction in insulin requirements (25-32), and, where measured, less hypoglycemia $(25,26,29)$. However, improvement in HbA1c has not consistently been demonstrated, despite effective C-peptide preservation. This inconsistency is perhaps not surprising in interventional studies where treatment groups are expected to achieve the same glycemic targets by intensifying insulin therapy. However, there are other factors that may explain this disconnect, including the importance of patient factors in insulin management and determining $\mathrm{HbA} 1 \mathrm{c}$ levels as well as the variation in timing of C-peptide loss between individuals.

Collectively, the data suggest that the relationship between HbA1c levels and C-peptide is different from the relationship with hypoglycemia. The former has a steep sigmoidal relationship (Figure 1), whereby at high C-peptide levels, changes in HbA1c levels are buffered by a sufficient cell response to high glucose levels alone with insulin adjustments by patients. But low levels of C-peptide are insufficient to affect hyperglycemia. By contrast, as illus- 
trated by Gubitosi-Klug et al. (4), even few functional $\beta$ cells can markedly affect hypoglycemia by virtue of being exquisitely responsive to declining glucose levels (22). The JDRF/Diabetes UK Trial Outcome Marker Initiative (TOMI; https:/c-path. org/programs/tomi-t1d/) will help clarify whether this analysis is correct; by collating data sets from multiple trials and observational studies, TOMI will explore the relationship of C-peptide to clinical variables and generate quantitative estimates of what enough C-peptide preservation might be in different populations and for different outcomes.

\section{Conclusions}

Insulin-induced hypoglycemia is the most frequent complication of intensive insulin therapy, and, therefore, the findings of Gubitosi-Klug et al. (4) suggest a clinical importance for management of patients and support maintenance of residual $\beta$ cell function as a goal for disease modification. To achieve improved HbA1c levels and, hence, reduce macrovascular complications, greater residual cell function may be needed. Nonetheless, it is important to remain anchored in the lessons from prediabetes and the early postdiagnosis period: preserving $\beta$ cell function is associated with easier glycemic control and more individuals achieving glycemic targets. Preserving this level or even the levels seen in prediabetes allows near-perfect glycemic control without the burden and risks of insulin therapy. As we approach 100 years of insulin therapy, patients and physicians should prioritize early and sustained intervention soon after T1D diagnosis or even in the preclinical stage (33). Patients that can preserve $\beta$ cell function at levels seen at the diagnosis of T1D, higher than those observed by Gubitosi-Klug et al. (4) (>0.2 nmol/L), with improved physiologic characteristics, may have optimal long-term benefit.

\section{Acknowledgments}

$\mathrm{KCH}$ is supported by grants DK057846, DK116577, DK085466, and TR001863 from the NIH and SRA-2019-833 from the Juvenile Diabetes Research Foundation.

Address correspondence to: Kevan C. Herold, Departments of Immunobiology and Internal Medicine, Yale University, 300
George St., \#353E, New Haven, Connecticut 06520, USA. Phone: 203.785.6507; Email: Kevan.herold@yale.edu.

1. Foster NC, et al. State of type 1 diabetes management and outcomes from the T1D exchange in 2016-2018. Diabetes Technol Ther. 2019;21(2):66-72

2. Nirantharakumar K, et al. Clinically meaningful and lasting HbA1c improvement rarely occurs after 5 years of type 1 diabetes: an argument for early, targeted and aggressive intervention following diagnosis. Diabetologia. 2018;61(5):1064-1070.

3. Hao W, et al. Fall in C-peptide during first 4 years from diagnosis of type 1 diabetes: variable relation to age, HbA1c, and insulin dose. Diabetes Care. 2016;39(10):1664-1670.

4. Gubitosi-Klug R, et al. Residual $\beta$ cell function in long-term type 1 diabetes associates with reduced incidence of hypoglycemia. J Clin Invest. 2021;131(3): 143011.

5. McGee P, et al. Insulin secretion measured by stimulated C-peptide in long-established Type 1 diabetes in the Diabetes Control and Complications Trial (DCCT) / Epidemiology of Diabetes Interventions and Complications (EDIC) cohort: a pilot study. Diabet Med.2014;31(10):1264-1268.

6. Steffes MW, et al. Beta-cell function and the development of diabetes-related complications in the diabetes control and complications trial. Diabetes Care. 2003;26(3):832-836.

7. Palmer JP, et al. C-peptide is the appropriate outcome measure for type 1 diabetes clinical trials to preserve beta-cell function: report of an ADA workshop, 21-22 October 2001. Diabetes. 2004;53(1):250-264.

8. Effect of intensive therapy on residual beta-cell function in patients with Type 1 diabetes in the Diabetes Control Complications Trial. Ann Intern Med.1998;128(7):517-23.

9. Steele C, et al. Insulin secretion in type 1 diabetes. Diabetes. 2004;53(2):426-433.

10. Balti EV, et al. Hyperglycemic clamp and oral glucose tolerance test for 3-year prediction of clinical onset in persistently autoantibody-positive offspring and siblings of type 1 diabetic patients. J Clin Endocrinol Metab. 2015;100(2):551-560.

11. Ferrannini E, et al. Progression to diabetes in relatives of type 1 diabetic patients: mechanisms and mode of onset. Diabetes. 2010;59(3):679-685.

12. Van Dalem A, et al. Relationship between glycaemic variability and hyperglycaemic clampderived functional variables in (impending) type 1 diabetes. Diabetologia. 2015;58(12):2753-2764.

13. Polonsky KS, et al. Abnormal patterns of insulin secretion in non-insulin-dependent diabetes mellitus. NEngl JMed. 1988;318(19):1231-1239.

14. Sosenko JM, et al. The influence of body mass index and age on C-peptide at the diagnosis of type 1 diabetes in children who participated in the diabetes prevention trial-type 1. Pediatr Diabetes. 2018;19(3):403-409.

15. Rickels MR, Robertson RP. Pancreatic islet transplantation in humans: recent progress and future directions. Endocr Rev. 2019;40(2):631-668.
16. Brooks AM, et al. Demonstration of an intrinsic relationship between endogenous C-peptide concentration and determinants of glycemic control in type 1 diabetes following islet transplantation. Diabetes Care. 2015;38(1):105-112.

17. Vantyghem MC, et al. Continuous glucose monitoring after islet transplantation in type 1 diabetes: an excellent graft function ( $\beta$-score greater than 7 ) Is required to abrogate hyperglycemia, whereas a minimal function is necessary to suppress severe hypoglycemia ( $\beta$-score greater than 3$)$. JClin Endocrinol Metab. 2012;97(11):E2078-E2083.

18. Buckingham B, et al. CGM-measured glucose values have a strong correlation with $\mathrm{C}$-peptide, $\mathrm{HbA1c}$ and IDAAC, but do poorly in predicting $\mathrm{C}$-peptide levels in the two years following onset of diabetes. Diabetologia. 2015;58(6):1167-1174.

19. Pinckney A, et al. Correlation among hypoglycemia, glycemic variability, and C-peptide preservation after alefacept therapy in patients with type 1 diabetes mellitus: analysis of data from the immune tolerance network T1DAL trial. Clin Ther. 2016;38(6):1327-1339.

20. Sorensen JS, et al. Residual $\beta$-cell function 3-6 years after onset of type 1 diabetes reduces risk of severe hypoglycemia in children and adolescents. Diabetes Care. 2013;36(11):3454-3459.

21. Nordwall M, Ludvigsson J. Clinical manifestations and beta cell function in Swedish diabetic children have remained unchanged during the last 25 years. Diabetes Metab Res Rev. 2008;24(6):472-479.

22. Rickels MR, et al. High residual C-peptide likely contributes to glycemic control in type 1 diabetes. J Clin Invest. 2020;130(4):1850-1862.

23. Marren SM, et al. Persistent C-peptide is associated with reduced hypoglycaemia but not $\mathrm{HbA1c}$ in adults with longstanding Type 1 diabetes: evidence for lack of intensive treatment in UK clinical practice? Diabet Med. 2019;36(9):1092-1099.

24. Gibb FW, et al. Preserved C-peptide secretion is associated with fewer low-glucose events and lower glucose variability on flash glucose monitoring in adults with type 1 diabetes. Diabetologia. 2020;63(5):906-914.

25. Ovalle F, et al. Verapamil and beta cell function in adults with recent-onset type 1 diabetes. Nat Med. 2018;24(8):1108-1112.

26. Rigby MR, et al. Alefacept provides sustained clinical and immunological effects in newonset type 1 diabetes patients. J Clin Invest. 2015;125(8):3285-3296.

27. Mastrandrea L, et al. Etanercept treatment in children with new-onset type 1 diabetes: pilot randomized, placebo-controlled, double-blind study. Diabetes Care. 2009;32(7):1244-1249.

28. Quattrin T, et al. Golimumab and beta-cell function in youth with new-onset type 1 diabetes. N Engl J Med. 2020;383(21):2007-2017.

29. Pescovitz MD, et al. Rituximab, B-lymphocyte depletion, and preservation of beta-cell function. N Engl J Med. 2009;361(22):2143-2152.

30. Orban T, et al. Co-stimulation modulation with abatacept in patients with recent-onset type 1 diabetes: a randomised, double-blind, placebo-controlled trial. Lancet. 2011;378(9789):412-419.

31. Herold KC, et al. Teplizumab (anti-CD3 mAb) 
treatment preserves $\mathrm{C}$-peptide responses in patients with new-onset type 1 diabetes in a randomized controlled trial: Metabolic and immunologic features at baseline identify a subgroup of responders. Diabetes. 2013;62(11):3766-3774.

32. Herold KC, et al. Anti-CD3 monoclonal antibody in new-onset type 1 diabetes mellitus. $\mathrm{NEnglJ}$
Med. 2002;346(22):1692-1698.

33. Herold KC, et al. An anti-CD3 antibody, teplizumab, in relatives at risk for type 1 diabetes. NEngl J Med. 2019;381(7):603-613. 